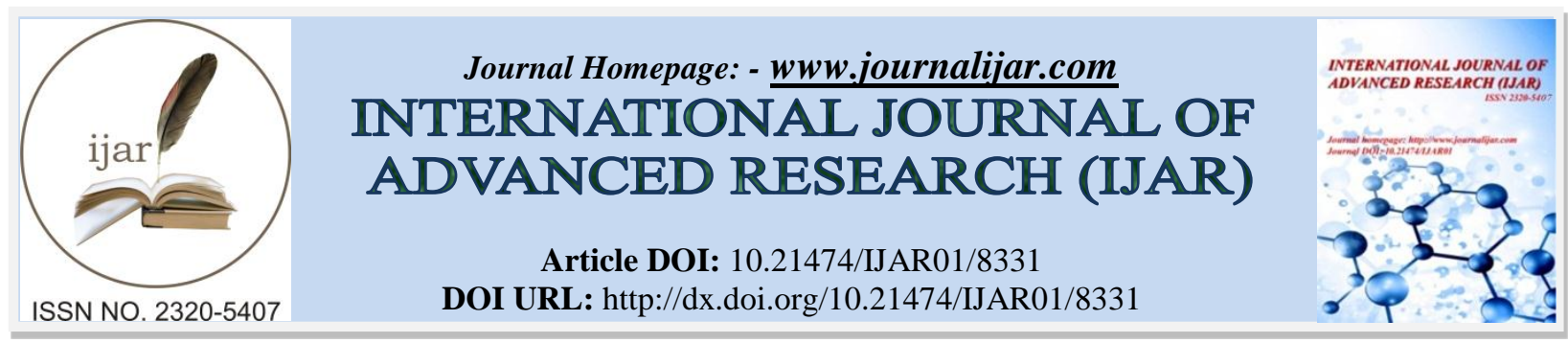

RESEARCH ARTICLE

\title{
A NOVEL APPROACH TO CLASSIFY AND CONVERT 1D SIGNAL TO 2D GRAYSCALE IMAGE IMPLEMENTING SUPPORT VECTOR MACHINE AND EMPIRICAL MODE DECOMPOSITION ALGORITHM.
}

M. Azad, F. Khaled and M.I. Pavel.

Department of Computer Science and Engineering, BRAC University, Dhaka, Bangladesh.

\section{Manuscript Info}

Manuscript History

Received: 08 November 2018

Final Accepted: 10 December 2018

Published: January 2019

Key words:-

Signal processing, Empirical mode decomposition, support vector machine, $1 \mathrm{D}$ to $2 \mathrm{D}$ conversation, segmentationbased fractal texture investigation.

\begin{abstract}
This paper represents a novel approach to transform one dimension (1D) signals into two dimension (2-D) grayscale image and a feature extraction process to extricate detail texture data of this $2 \mathrm{D}$ image to classify signals utilizing multi-class support vector machine. In all previous approaches of the signal processing strategies, the signal is continuously processed in one dimension (1-D) representation. Hence, a gigantic relationship information between time and frequency coefficients is effectively missing. To annihilate these issues, two dimensions representation of the signal is assessed in this paper. Centering on creating a proficient highlight extraction strategy for evacuating deficiencies of motor signals utilizing the 2-D image. Each pixel is taken and squaring it to discover out the energy and making it to gray image. The esteem of tests is normalized based on the tests of the signals within the time space, and Empirical Mode Decomposition (EMD) is to distinguish the low frequency which fundamentally represents to noise and evacuate it from the image. Segmentation-based Fractal Texture Investigation (SFTA) algorithm is used to extricate the feature vectors which are utilized for classifying the signals using multi-class support vector machine (SVM). The precision is $88.57 \%$ which is picked up from confusion matrix while classifying signals.
\end{abstract}

Copy Right, IJAR, 2018,. All rights reserved.

\section{Introduction:-}

The field of signal processing is a very important field of study and one that makes possible various other fields such as communications. MP3 music files contain processed, transformed, and compressed music signal data. Speech recognition systems such as dictation software need to analyze and process signal data to identify individual words in a spoken sentence. Neural interface devices, such as various medical prostheses must read the complicated signals of neurons, process those signals to determine the important features, and then convert those features to digital data. Signal processing enables high-speed data communication, even in the presence of interference or noise [2,13]. We all want to see a perfect image. The view of on image depends on the good quality of the image and the good quality refers to the perfect texture combination. So by representing texture in a perfect way we can easily make a perfect image. And it is a must for the viewers to see a good quality image. Basically our motive is to create a perfect good quality image which will be useful in business sectors and in the industries to detect the basic elements with clarity. For a developing country like us there are many industries where productivity depends on the reliability and

Corresponding Author:-M. Azad.

Address:-Department of Computer Science and Engineering, BRAC University, Dhaka, Bangladesh. 
efficiency of the machines, motors. In order to monitor if they are working without any disturbance or not their signals need to be checked. For this kind of reason image processing from signal and their feature extraction has become very popular subject in a modern era like this. In recent years two dimensional (2D) representation of 1D signal has been used in a variety of sectors. These images can be processed and features are extracted which plays an important role in many applications which shows the significance of the process of conversion of 1D signal to 2D image and feature extraction from it. While doing it people faced many difficulties and complexities as they went to define significant features of the texture [18].2D representation from 1D signals has been widely researched and came out with desired results but the feature extraction of texture images is still in research. The drawbacks of these process has motivated us to come up with a more efficient method of $2 \mathrm{D}$ representation of $1 \mathrm{D}$ signal and feature extraction of the texture image [3]. In our proposed feature extraction method for 2D gray-level image at first we convert the 1D signal into 2D gray-level image, essential pixels counting due to generating the feature vector using SFTA, and then train the classifier by applying the Support Vector Machine. In this proposed system, the samples of a signal are normalized into the range 0-255 of a gray image and assembling into the 2D space, forming an image. Our efficient method is to take the energy of the pixels and make a 2D gray-level image. Finally compare it with the other feature extraction approaches by providing the accuracy [5].

\section{Proposed Methodology:-}

The diagram in Figure 1 shows the detailed demonstration of our proposed model and how the algorithm is going to work to efficiently extract features and detect their classes. At first we collected we wave signals for our experiment .After applying empirical mode decomposition we found different IMF's from the signal and from those IMF's we cut off few lower IMF's of lower frequencies which made our signal smoother. Then we calculated the energy of our signals which we have used for converting into 2-D images. Then converted the energy spectrum of the signal into 2-D image. Followed by converting the signals into 2-D we extracted features of the 2-D images using STFA algorithm in which we segmented the images into sets of binary images. We measured 21 features for each image of our training dataset. We have used SVM classifier for the purpose of classification of our images as it is one of the efficient and accurate classifiers. The feature vectors were used to train the classifier SVM. The trained classifier was able to efficiently detect our test images and label their class.

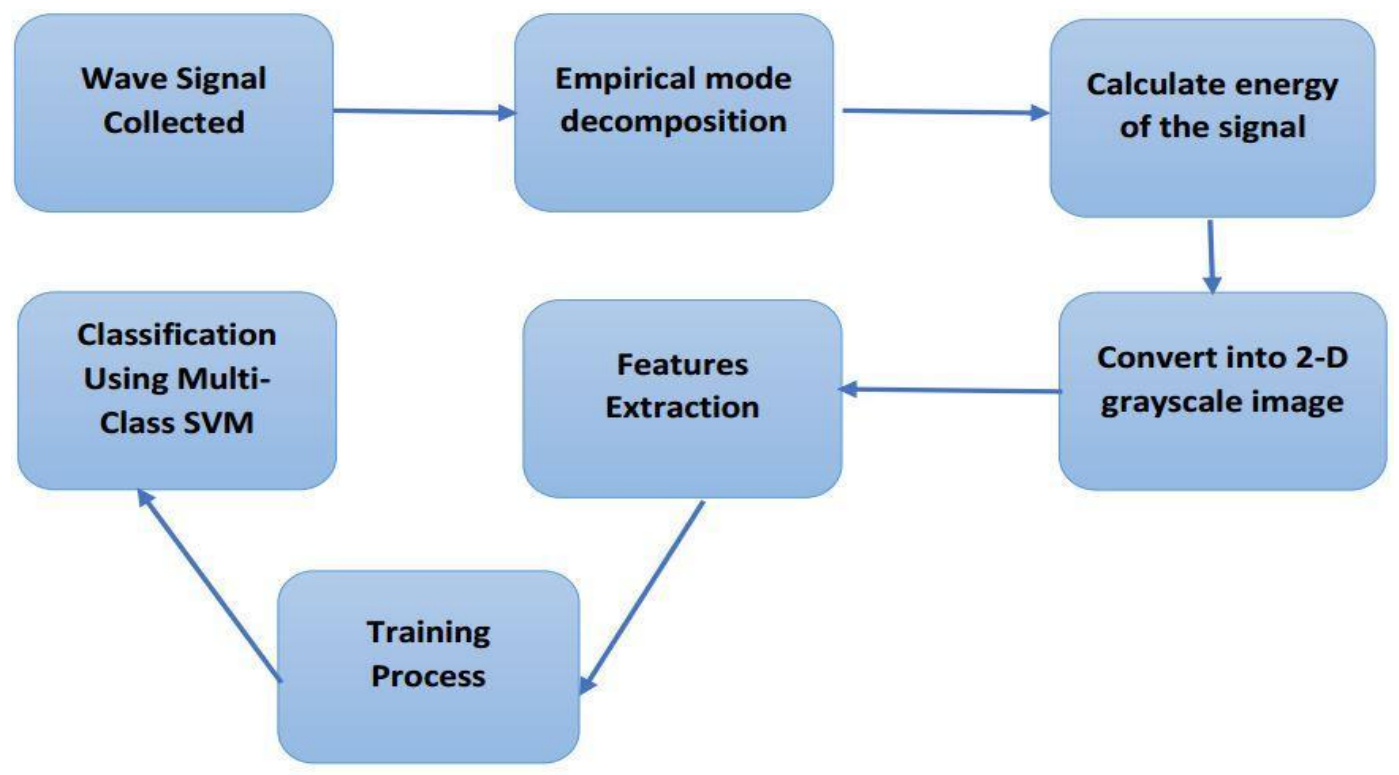

Figure 1:- Block diagram of proposed methodology

\section{Wave Signal:}

Dataset: In our experiment we have mainly collected wave signal of the faulty of the industrial motors. Motors are used to simulate the fault condition such as broken rotor bar fault (BRBF), rotor imbalance fault (RIF), bowed shaft fault (BSF), bearing fault (BF), phase imbalance fault (PIF), angular misalignment fault (AMF), Parallel Misalignment Fault (PMF) [4].From these conditions wave signals were collected which depicts different nature of 
the signal. The signal were samples at $8 \mathrm{kz}$ frequencies and one second long in duration. The different fault conditions were as below:

Table 1:- Fault conditions of dataset

\begin{tabular}{|c|c|}
\hline Fault condition & Properties \\
\hline The Broken Rotor Fault & These 12 of 34 rotor bars have been broken \\
\hline Rotor Imbalance Fault & These masses $(8.4 \mathrm{~g})$ were added into one side of rotor. \\
\hline Bowed Shaft Fault & The shaft has been bowed $0.075 \mathrm{~mm}$ \\
\hline Bearing Fault & The out raceway has been damaged \\
\hline Phase Imbalance Fault & One phase has been adjusted by adding $4.30 \Omega$ resistor \\
\hline Angular Misalignment Fault & The bearing pedestal has been adjusted $0.480 \Omega$ \\
\hline Parallel Misalignment fault & The counterbalance between two center lines of the motor and load has been \\
& converted $0.1 \mathrm{~mm}$
\end{tabular}

\section{Empirical mode decomposition (EMD):}

After the wave signals data have been accumulated for different kind of condition, we associated correct mode breaking down figuring on those signals. EMD estimation basically disintegrates signs into a series frequency ordered IMF [6]. Every dynamic IMF contains cut down repeat movements than the past one. After performing EMD algorithm having found out different IMF for an input signal we ignored few of the lowest frequency IMF's of the signal and created a refined signal having only higher frequencies as Characteristics of signal. This movement of work helped us to improve the idea of the input signal and subsequently give purposes of enthusiasm for the later system of the experiment [15].
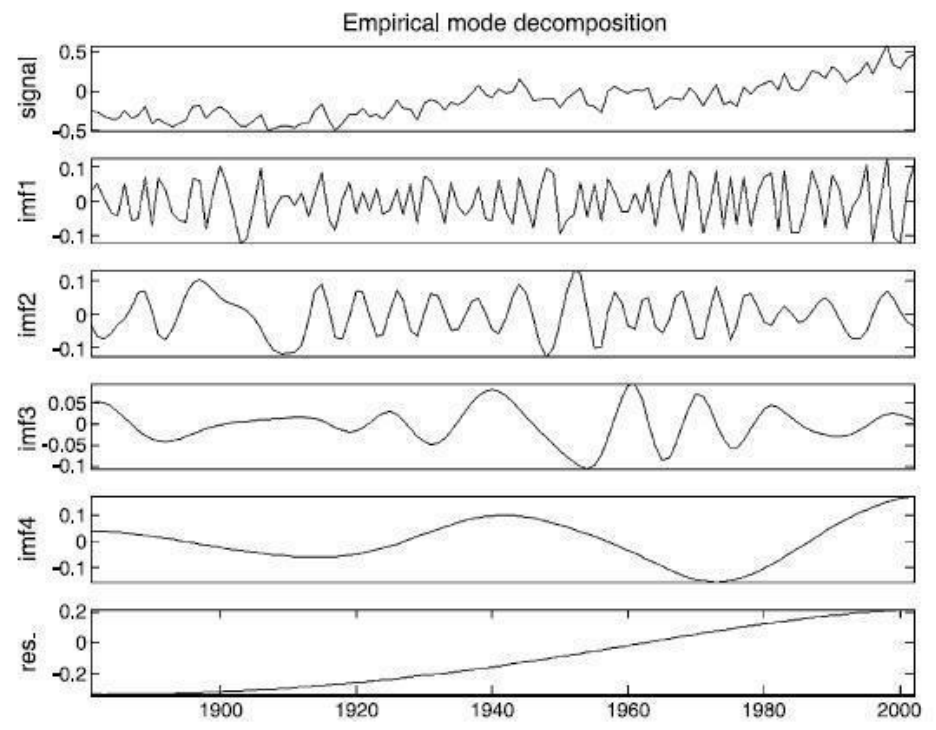

Figure 2:- Decomposition of signal into series of IMF's [6].

The figure above demonstrates that the progressive IMF's frequencies are lower than the past one. The most minimal one is known as residue. We expelled few lower IMF's alongside the residue to enhance the nature of our input signal. Energy is one of the imperative highlights of a signal. In our experiment we compute energy of the signal utilizing the following formula:

$$
E=\int_{-\infty}^{\infty}|x(t)|^{2} d t
$$

Instead of using the numerical values of the time domain of the signal we have used the energy of the wave signal which help use to depict the nature of the signal more efficiently. Using the energy values of the signal in the next stage we have converted the signal into 2-D image.

\section{Convert into 2-D gray-scale image:}

In this procedure, the vibration signals which are in time space, have been changed over into 2-D dimension utilizing the energy esteems. Toward the starting, the signals are fragmented into equivalent subparts each known as 
a frame The extent of the frame is figured as the multiplication of the frame duration and frequency rate of the signal. After segmenting the signals, we got a definite number of frames which along with the frame size helped to define the height and width of the matrix The height of the framework is equivalent to the frame size and the width of the matrix is equivalent to the quantity of the frames [4].For occasion, if our frame size is $\mathrm{X}$ and number of the frame is $\mathrm{Y}$ then our size of the desired matrix is $\mathrm{X}^{*} \mathrm{Y}$. Afterward, the energy estimations of the edges are put into the cells of the matrix. The estimation of each edge is moved into the grid vertically. The energy values of first frame is put into the first column of the matrix as the first value goes into the first cell of the first row, second energy value goes into the first cell of the second row and so on. As a result, the last value of the first frame will be put into the first cell of the last column as the height of the matrix is equal to the size of the frame thus every value fits into the column. Also all other frame energy esteems are put into the matrix consecutively one by one. As the width of the matrix is equal to the number of frames all the frames can be fit in the matrix. After all the values have been set into the matrix we now normalized the values of the frames in the range of 0-255.The lowest numerical value of the matrix is considered as 0 and the highest value is considered as 255.Considering this range all the numerical qualities are figured according to their proportions. These new values at that point supplant the old values in the matrix. These new values then replace the old values in the matrix. Normalization step helps to decrease the resolution of the original signal. This also helps to minimize noise on the acquired signals.2-D representation maintains features of signal in time domain and also texture feature of the signal in 2-D could be extracted to detect the signal.

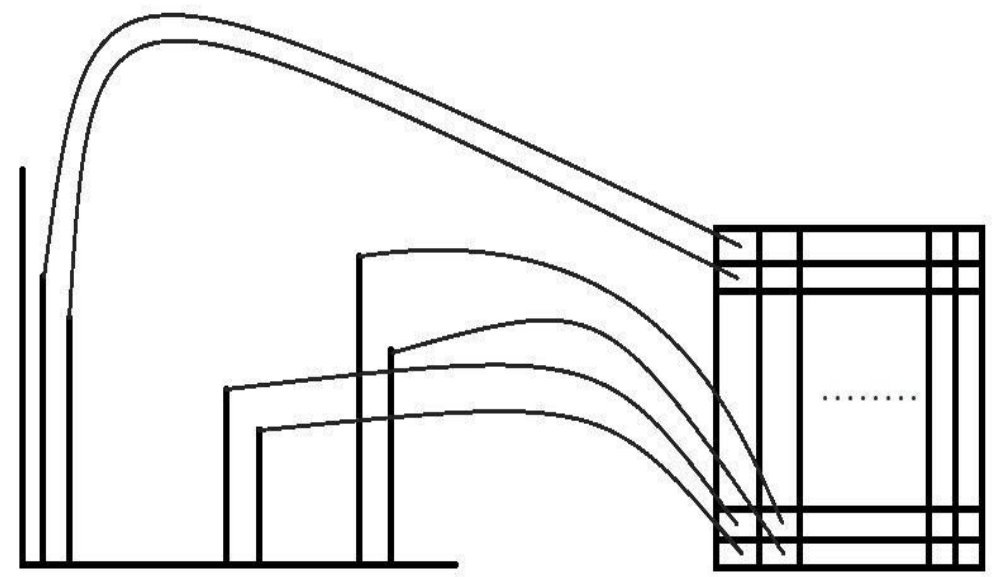

$$
12 \ldots \ldots \ldots(\mathrm{X}-1) \mathrm{X} \ldots(\mathrm{XX}-1) 2 \mathrm{X}
$$

(a) (b)

Figure 2:- Conversation of matrix (b), using the energy values (a) of the sample.

We have also used Frequency domain of signal which is representing the signal as a pair of amplitude and phase values at each component frequency. By using the frequency domain of the signal we also converted it into 2D.Before that the vibration signal is changed into frequency domain by applying Fourier Transform. As we created 2-D representation using time domain coefficients similarly we created 2-D representation using frequency domain coefficients and followed similar process like the previous one. After that we normalized the values of the signal in the range of 0-255. However frequency domain 2-D representation did not produce any significant texture for different kinds of signals as almost all signal's efficient pattern converges in the low frequency band.

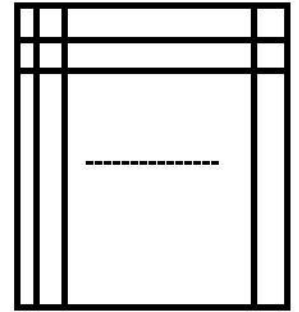

(a)

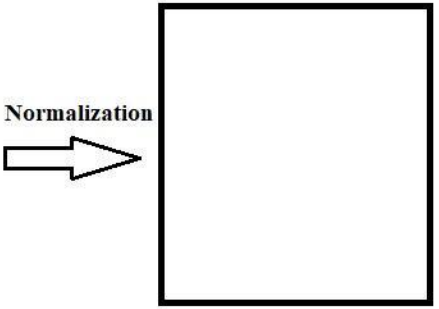

(b)

Figure 3:- Producing 2D gray Scale image (b), from matrix (a) 


\section{Features Extraction from 2-D Image:}

Further, the conversion of input signal into 2-D image is completed, at that point, feature vectors are extracted from the converted images using SFTA algorithm [8-10]. In this progression, we fragment our 2D image into a set of binary images utilizing the two threshold binary decomposition algorithm [10]. From the binary images, SFTA include vector will be processed by getting the values of the image size, mean gray level and boundaries fractal dimension. The element vector's size is three times of the number of the binary images as we have three components for each binary images which are image size, mean gray level and boundaries fractal dimension. For the experimental purpose, binary images for each input image, as a result the size of our feature row vector is 21.

\section{Training Classifier:}

We have extracted features from converted 2-D images, then multi-class support vector machine [22,23] algorithm is implemented using Matlab to classify the input signals. Feature vectors are used as our training data to train the classifier. 20 signals have been taken for having feature row vector of size 21 therefore our feature matrix has size of $20 * 21$. To spelt the training dataset from test, we have used $80 \%$ of our dataset for training purpose of the classifier and rest $20 \%$ of data are set used for testing. In the training process, we have trained the classifier by using label true and false with testing dataset class which helped us finding our result after the experiment was done. After completion of training the classifier, we tested rest of the dataset for classifying the classes of the signal. The feature vector of the input files' 2-D images were used to classify classes comparing to the training sets feature vectors. After classifying the SVM classifier gave output as 'True' or 'False' label. After we got result for testing dataset, confusion matrix is generated for measuring accuracy of our experiment to ensure the efficiency of the above mentioned approach [19].

\section{Result And Analysis:-}

In our test, we have collected flawed signals from mechanical motor. Observational mode deterioration is connected on the signals to enhance the quality of the signals. Prior to that we have calculated the energy values of the signals and utilized to convert into 2-D gray-scale image. At that point, we utilized the gray scale image to extract features using SFTA algorithm. The training data features are being utilized to prepare classifier. Furthermore, following a comparable process and extracted feature from testing data set which was utilized to classify. We have utilized confusion matrix to measure our classification precision. In this following research, we have basically collected wave signal of the flawed condition of the mechanical motors. Motors are utilized to reenact the fault conditions such as Broken Rotor Bar Fault(BRBF), Rotor Imbalance Fault(RIF),Bowed Shaft Fault(BSF),Bearing Fault(BF),Phase Imbalance fault(PIF),Angular Misalignment Fault(AMF),Parallel Misalignment Fault(PMF). From these conditions wave signals were collected which portrays diverse nature of the signal. The signal were tests at $8 \mathrm{kz}$ frequencies and one moment long in length. Presently, EMD algorithm is applied on the signals to enhance the quality of the signals by breaking down the signal into diverse IMF's. Each progressive IMF has lower recurrence level than the past one. We have evacuated few of the lower IMF's to form the signal smoother and solid to appear distinctive design for distinctive sort of signals. After performing EMD calculation we have calculated the energy values of the signals by utilizing energy equation condition. Utilizing the energy values we have changed over the signal into 2-D gray-scale image. Matlab is used for our transformation strategy of signal to 2-D gray-scale image. Signals are then partitioned the signal into a number of outlines. Each frame's energy value was put into matrix vertically sequentially. The matrix height was defined by the size of the frame and matrix width was defined by the number of the frame. After all the values were put into the matrix Matlab function was used to plot the 2-D image of the input signals energy. The signal's numerical value, mean grayscale value of neighbor pixels and discrete fourier transform of a sequence calculated by applying fast fourier transform [14] are transformed to create the 2-D image and compared the image with energy value signal's image. In the following figures the comparison can be observed: 


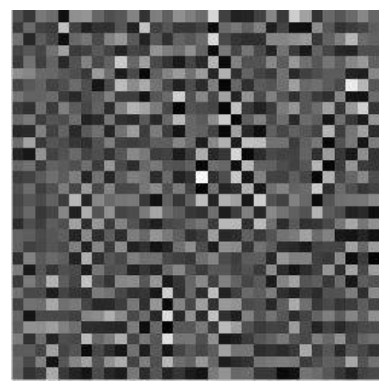

(a)

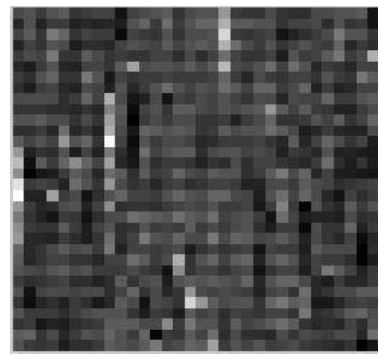

(e)

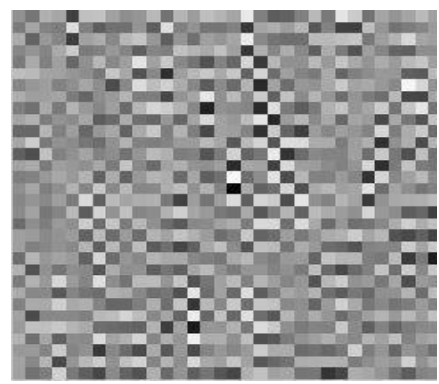

(b)

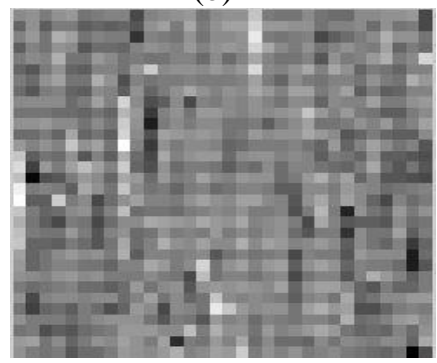

(f)

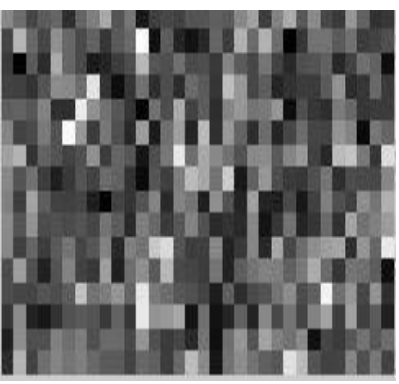

(c)

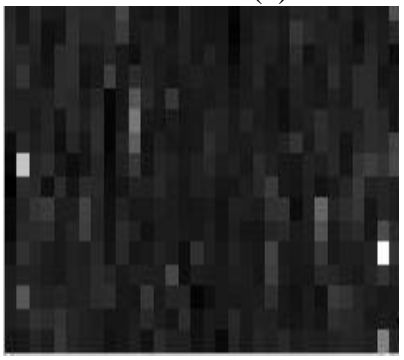

(g)

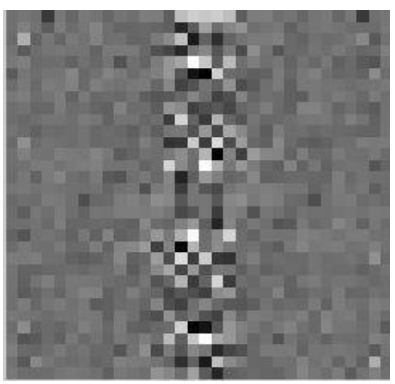

(d)

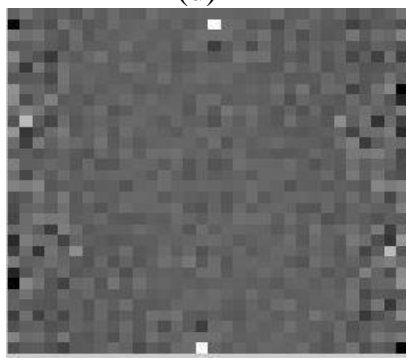

(h)

Figure 4:- 2-D image using $(a, e)$ short term energy , $(b, f)$ numerical value , $(c, g)$ mean value of the fourier gray scale value,$(\mathrm{d}, \mathrm{h})$ fourier transform

In Figure 4.a, 4.b, 4.c, 4.d , 2-D images of bearing fault signal can be observed which were obtained by short term energy values, signal numerical values, mean pixel values and fast fourier transform. In Figure 4.e, 4.f, 4.g, 4.h comparis among 2-D images of bowed shaft fault signal can be observed which were obtained by similar process. The best image texture pattern was found using the short term energy values of signal.

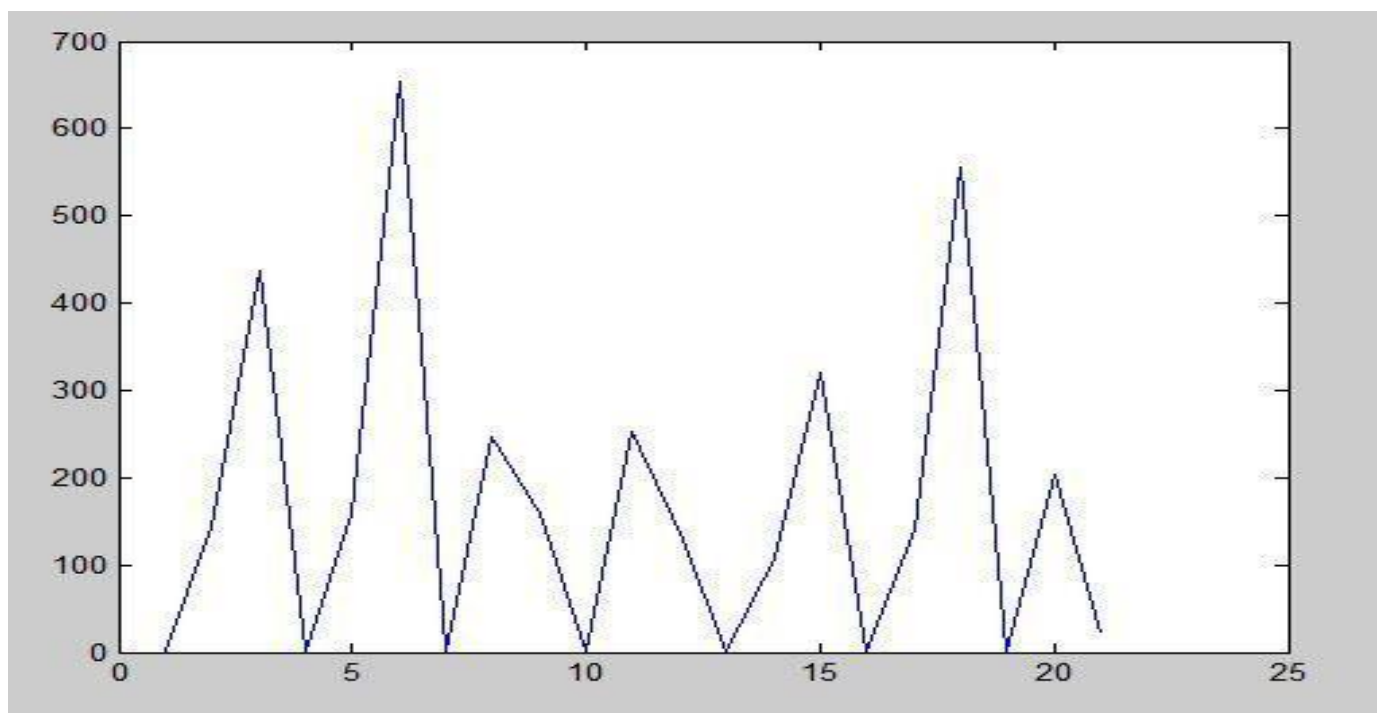

Figure 5:- Diagram of feature vector obtained from gray scale image

Using the feature vector of the training images we have trained the binary SVM classifier. We have used the images of bearing fault and bowed shaft fault to train the SVM classifier. After training the SVM classifier we have produced images for the test dataset of the bearing fault and bowed shaft fault. Then we used the test images for SVM to classify the image and label the class as 'true' or 'false'.

In the experiment we assumed, 
Bearing fault $=$ True

Bowed shaft fault $=$ False

After the classification process we have used confusion matrix to measure our classification accuracy to prove the efficiency of our approach for 2-D image feature extraction.

Table 2:- Confusion matrix to measure accuracy

\begin{tabular}{|l|l|l|l|}
\hline N=105 & Predicted False & Predicted True & Total \\
\hline Actual False & 40 & 5 & 45 \\
\hline Actual True & 7 & 53 & 60 \\
\hline & 47 & 58 & \\
\hline
\end{tabular}

$$
\begin{aligned}
\text { Accuracy } & =\left\{\frac{(40+53)}{40+5+7+53} * 100\right\} \\
& =88.57 \%
\end{aligned}
$$

The above mentioned accuracy shows that the SVM classifier was able to classify the test image class efficiently using the experiments approach of using energy values of the signal.

\section{Conclusion:-}

In this paper, we are proposing an efficient feature extraction method for 2D digital image. Our proposed approach is to take the energy of every pixel and put it on the matrix. At the beginning, the signals are segmented into equal subparts each known as a frame. The size of the frame is calculated as the multiplication of the frame duration and frequency rate of the signal. After segmenting the signal we got a definite number of frames which along with the frame size helped to define the height and width of the matrix. The height of the matrix is equal to the size of the frame size and the width of the matrix is equal to the number of the frames.

\section{Future Work:-}

We have a vision to take this research into another level and make it useful and profitable in business sectors. Hundreds of thousands of photographs are uploaded to the internet every minute through various social networking and photo sharing platforms. While some images get millions of views, others are completely ignored. Even from the same users, different photographs receive different number of views. This begs the question: What makes a photograph popular? Can we predict the number of views a photograph will receive even before it is uploaded? Two key components are investigated: the image content and social context. The main task of ours will be to remove the faults of an image to make it better and thus the photo will be viewed more. In future we also want to add an object classifier. An object classifier (based on Deep Learning) able to recognize 1000 object classes, is used to detect whether a given object class is present or not in the image. We also want to check the image Memorability in future and will measure it with a memory game on Amazon Mechanical Turks.

\section{Reference:-}

1. V. T. Do and U.-P. Chong, "Signal Model-Based Fault Detection and Diagnosis for Induction Motors Using Features of Vibration Signal in Two-Dimension Domain," Strojniški vestnik - Journal of Mechanical Engineering, vol. 57, no. 09, pp. 655-666, 2011.

2. Zhou, R., "Feature extraction of musical content for automatic music transcription.", EPFL scientific publications, 2006. doi: 10.5075/epfl-thesis-3638

3. F. M. Khellah, "Texture Classification using Dominant Neighborhood Structure”. IEEE Trans, Image Process. vol. 20 (11), pp. 3270-3279, 2011.

4. Chang, T., Kuo, C. C., "Texture analysis and classification with tree-structured wavelet transform" IEEE Transactions on image processing, 2(4), 429-441, 1993.

5. Dinh Nguyen Van, "Two Dimension Representation Approach for Developing the Fault Diagnosing System based on Vibration Signal". University of Ulsan, Republic of Korea, 2013.

6. Lei, Y., Lin, J., He, Z., \& Zuo, M. J. , “A review on empirical mode decomposition in fault diagnosis of rotating machinery." Mechanical Systems and Signal Processing, 35(1-2), 108-126, 2013.

7. P. Liao, T. Chen, and P. Chung, "A Fast Algorithm for Multilevel Thresholding," Journal of Information Science and Engineering, vol. 17,no. 5, pp. 713-727, 2001. 
8. Costa, A. F., Humpire-Mamani, G., Traina, A. J. M. , “An efficient algorithm for fractal analysis of textures.” In Graphics, Patterns and Images (SIBGRAPI), 25th SIBGRAPI Conference, pp. 39-46, 2012.

9. Balan, A. G., Traina, A. J., Traina, C., Azevedo-Marques, P. M., "Fractal analysis of image textures for indexing and retrieval by content." In Computer-Based Medical Systems, Proceedings. 18th IEEE Symposium on pp. 581-586, 2005.

10. D. Saraswathi, G. Sharmila and E. Srinivasan, "An automated diagnosis system using wavelet based SFTA texture features," International Conference on Information Communication and Embedded Systems (ICICES2014), Chennai, pp. 1-5, 2004. doi: 10.1109/ICICES.2014.7034123

11. X. Wu, V. Kumar, J.R. Quinlan, J. Ghosh, Q. Yang, H. Motoda, G.J. McLachlan, N. Angus, B. Liu, P.S. Yu, Z.H. Zhou, M. Steinbach, D.J. Hand, D. Steinberg, "Top 10 algorithms in data mining." Journal of knowledge and information systems, Vol. 14 (1), pp. 1-37, 2007.

12. V. N. Vapnik. "Statistica $0 \mathrm{ml}$ learning theory. John", Wiley\& Sons, New York, 1998.

13. R. M. Haralick, K. Shanmugam, and I. H. Dinstein, "Textural Features for Image Classification," IEEE Transactions on Systems, Man and Cybernetics, vol. 3, no. 6, pp. 610-621, 1973.

14. R. J. Polge , B. K. Bhagavan, "Efficient Fast Fourier Transform Programs for Arbitary Factors with One Step Loop Unscrambling", IEEE Transactions on Computers, v.25 n.5, p.534-539, May 1976.

15. Huang, N. E., Shen, Z., Long, S. R., Wu, M. C., Sih, H. H., Zheng, Q., Liu, H. H., "The empirical mode decomposition and the Hilbert spectrum for nonlinear and non-stationary time series analysis". In Proceedings of the Royal Society of London A: mathematical, physical and engineering sciences, vol. 454, no. 1971, pp. 903-995, 1998.

16. Rilling, G., Flandrin, P., Goncalves, P., "On empirical mode decomposition and its algorithms" In IEEEEURASIP workshop on nonlinear signal and image processing, Vol. 3, pp. 8-11,NSIP-03, Grado (I), 2003.

17. Phinyomark, A., Phukpattaranont, P., Limsakul, C. (2012), "Feature reduction and selection for EMG signal classification. Expert Systems with Applications", 39(8), 7420-7431.

18. Liu, Z., Wang, Y., \& Chen, T., "Audio feature extraction and analysis for scene segmentation and classification." Journal of VLSI signal processing systems for signal, image and video technology, 20(1-2), pp. 61-79, 1998.

19. Jung, K., Kim, K. I., \& Jain, A. K., "Text information extraction in images and video: a survey. Pattern recognition," 37(5), 977-997, 2004.

20. Deng, X., Liu, Q., Deng, Y., Mahadevan, S., "An improved method to construct basic probability assignment based on the confusion matrix for classification problem." Information Sciences, 340, pp. 250-261, 2016.

21. P. Tian, J. Wang, W. Zhang and J. Liu, "A Fault Tree Analysis Based Software System Reliability Allocation Using Genetic Algorithm Optimization," WRI World Congress on Software Engineering, Xiamen, 2009, pp. 194-198, 2009. doi: 10.1109/WCSE.2009.227.

22. M. A. Hearst, S. T. Dumais, E. Osuna, J. Platt and B. Scholkopf, "Support vector machines," in IEEE Intelligent Systems and their Applications, vol. 13, no. 4, pp. 18-28, July-Aug. 1998. doi: 10.1109/5254.708428

23. G. M. Foody and A. Mathur, "A relative evaluation of multiclass image classification by support vector machines," in IEEE Transactions on Geoscience and Remote Sensing, vol. 42, no. 6, pp. 1335-1343, June 2004. doi: 10.1109/TGRS.2004.827257. 